\title{
Efectividad de la rehabilitación vestibular en una serie clínica
}

\author{
Effectiveness of vestibular rehabilitation in a clinical series
}

Héctor Riveros', Caudio Correa G, José L. Anabalón B², Carolina Aranís J3.

\section{RESUMEN}

Introducción: La rehabilitación vestibular (RV) consiste en ejercicios que aceleran la compensación laberíntica, aumentando y/o mejorando la actividad de los otros dos sistemas que intervienen en el sistema del equilibrio.

Objetivo: Evaluar la efectividad de un programa de RV en pacientes de nuestro servicio de Corrinolaringología, con inestabilidad en la marcha secundaria a patología vestibular.

Material y método: Estudio prospectivo de pacientes con desequilibrio objetivado mediante examen de VIII par. Se les aplicó una encuesta de RV valorada para determinar el grado de desequilibrio y la alteración de actividades de la vida diaria Al grupo estudiado se aplicaron ejercicios de RV durante 6 semanas, y el grupo control recibió tratamiento farmacológico estándar.

Resultados: Se reclutaron 48 pacientes; 26 en el grupo estudiado y 22 en el grupo control. En el grupo en estudio, a las 6 semanas todos presentaron algún grado de mejoría, encontrándose sólo desequilibrio leve en 92\% y mejoría total en $8 \%$. En el grupo control, a las 6 semanas la sintomatología persistió en $50 \%$ de los pacientes y el $50 \%$ restante presentó aún desequilibrio leve; ninguno se recuperó totalmente.

Discusión: La RV es un programa de ejercicios de aplicación no invasiva, de fácil realización en el hogar, que ha demostrado ser efectiva en la disminución de la inestabilidad, con lo cual se recobran gran parte de las actividades del diario vivir.

Palabras clave: Vértigo, rehabilitación vestibular, sistema propioceptivo y ocular.

\begin{abstract} that participate in balance. group received standard pharmacological treatment.
\end{abstract}

Introduction: Vestibular rehabilitation (VR) consists of a series of exercises that increase labyrinthine compensation and/or improve the activity of the other two systems

Aim: To evaluate the effectiveness of a VR program in patients in our ORL department presenting with walking instability secondary to vestibular pathology.

Material and method: Prospective study of patients with objective imbalance as demonstrated by VIII nerve test. Patients were asked to answer a questionnaire on VR in order to determine the degree of imbalance and the level of daily life disturbances. The study group was assigned to a VR exercise program for 6 weeks, while the control

Results: 48 patients were included, 26 in the study group and 22 in the control group. In the study group, after 6 weeks all patients showed some degree of symptoms

\footnotetext{
Médico, Departamento de Otorrinolaringología de la Pontificia Universidad Católica de Chile.

Tecnólogo Médico ORL.

Interna de Medicina de la Pontificia Universidad Católica de Chile.
} 
improvement, with 92\% reporting only mild imbalance, and with complete rehabilitation in $8 \%$. In the control group, after 6 weeks symptoms persisted in $50 \%$ of patients, and the remaining $50 \%$ reported mild imbalance; none recovered completely.

Discussion: VR is a non-invasive exercise program, easily performed at home, that has demonstrated to be effective in reducing imbalance, which in turns results in that many of the daily activities can be performed again.

Key words: Vertigo, vestibular rehabilitation, proprioceptive and ocular systems.

\section{INTRODUCCIÓN}

日 equilibrio se logra mediante las aferencias de tres sistemas principales: el vestibular, el visual y el propioceptivo, los que se integran a nivel del Sistema Nervioso Central (SNC). 日 sistema vestibular es parte del oído interno, por lo que ambos vestíbulos deben funcionar en perfecta sincronía para que en el cerebro, cerebelo y tronco cerebral existaintegración. Así, la alteración del arco reflejo vestíbulo-óculo-motor produce vértigo periférico, definido como sensación de rotación de los objetos o del cuerpo en el espacio acompañado de inestabilidad en la marcha generalmente hacia el mismo lado del oído dañado y síntomas neurovegetativos; puede ser secundario a hipofunción, hiperfunción o ausencia de respuesta de los vestíbulos en el mayor porcentaje de los casos y/o de los músculos oculares y de las aferencias propioceptivas que llegan desde extremidades y columna'. En semanas, esta sintomatología va cediendo, puesto se produce una compensación vestibular, o recuperación funcional del equilibrio, paralo cual se necesita el reajuste del reflejo vestíbulo-oculomotor, del equilibrio estático y del equilibrio dinámico entre ambos complejos nucleares vestibulares, proceso llamado compensación vestibular. Paraesto, ocurrela reorganización de los circuitos neuronales de numerosas estructuras, como el cerebelo y tronco cerebral, para lograr finalmente un nuevo estado de equilibrio, donde no se recupera la lesión vestibular periférica.

日 vértigo de origen periférico es el más frecuente, y corresponde al $44 \%$ de los síndromes vertiginosos según Kroenke y cols ${ }^{2}$, en el año 2000. Tiene una elevada prevalencia en la población general, alcanzando en España, por ejemplo, una prevalencia del $5 \%$ al $10 \%$, con variación según grupos etarios ${ }^{3}$. También se describe que 1 de cada 3 adultos mayores presenta vértigo en su vida $^{4,5}$ y de los pacientes que sufren caídas crónicas, el $50 \%$ tendría desórdenes vestibulares ${ }^{6}$.
日 tratamiento en la fase aguda generalmente incluye drogas que inhiben las entradas sensoriales, las que en su mayoría tienen efecto sedante, causando una adaptación más lenta, demorando la respuestaa la terapia7. En la mayoría de los adultos jóvenes la compensación suele ocurrir de manera espontánea y rápida, pero en muchos de los que padecen enfermedades como parálisis cócleo vestibular súbita, paresia vestibular no compensada, laberintitis y neurolúes, entre otras, los síntomas de disfunción vestibular pueden ser persistentes, causando inestabilidad y desequilibrio que los incapacita crónicamente, impidiéndoles desarrollar su vida en forma normal, siendo éstos pacientes los candidatos para realizar un programa de rehabilitación vestibular (RV).

La RV es un buen recurso terapéutico, con éxito en el manejo de la inestabilidad de la marcha secundaria a una falla vestibular, siendo un buen complemento para lograr una acomodación vestibular efectiva ${ }^{8}$. Eimina los síntomas, disminuye la inestabilidad y el riesgo de caídas e incorpora al paciente a sus actividades habituales prontamente, acelerando el fenómeno de «recuperación funcional del equilibrio». Los conceptos iniciales de rehabilitación vestibular se establecen en la década de los años 40 por Cawthorne $^{9}$ y Cooksey ${ }^{10}$. En 1952 Dix y Hallpike ${ }^{11}$ sugieren a la rehabilitación vestibular como tratamiento del vértigo. En 1974 Hecker $^{12}$ reafirma y perfecciona el sistema de Cawthorne. Cohen ${ }^{13}$ en 1991 da a conocer un programa de prueba de la postura corporal. Whitney y cols ${ }^{14}$ mencionó la importancia de conocer la incapacidad de realizar las actividades de la vida diaria del paciente, y paralela mente Jacobson et $a^{15}$ en el año 1990, desarrolló una encuesta destinada a conocer los problemas presentados en el quehacer diario, encontrando proporcionalidad con la severidad de la fall a vestibular. En 1994 Foster y cols ${ }^{16}$ reafirma el beneficio de la rehabilita ción vestibular, y en 1995 se propone aplicar esta terapia en los lugares de trabajo ${ }^{17}$. 
La rehabilitación se fundamenta en el fenómeno fisiológico de compensación vestibular. Comprende ejercicios motores, oculares y vestibulares, los cuales desencadenan el cuadro de vértigo que afecta al paciente, hastaqueatravés de la habituación, logran la adaptación. También activan receptores periféricos del equilibrio, poniendo en marcha el proceso de sustitución sensorial, en los que adoptan nuevas estrategias para remplazar la función vestibular disminuida, contribuyendo a su vez a la recuperación de la estabilidad estática mediante la potenciación del reflejo cérvicooculomotor. Así se estimulan áreas del SNC no afecta das, mediante actividades y ejercicios repetitivos, los que a través de un proceso central voluntario de habituación llevan a la adaptación, logrando compensar y/o adaptar el mensaje asimétrico que envía el sistema vestibular.

Se describe en diversos estudios que sobre $80 \%$ de los pacientes se recuperarían ${ }^{18}$, porque existen factores que pueden afectar los resultados, como la edad del paciente, puesto que después de los 60 años la compensación vestibular es más lenta, logrando así resultados menos satisfactorios. También influirían en los resultados patologías crónicas como la diabetes mellitus y la hipertensión arterial y las secuelas de traumas y de intoxicaciones ${ }^{19}$.

Dado quela RV es un tratamiento alternativo quese ha utilizado en otros países como Inglaterra y Estados Unidos, con buenos resultados, pero que no se ha incorporado como tratamiento formal en los centros de nuestro país, principalmentedebido al desconocimiento de su existencia, queremos evaluar el grado de mejoría obtenido luego de la realización de un programa de RV en pacientes con trastornos del equilibrio producto de una falla vestibular periférica de nuestro Servicio de Corrinolaringología de la Red de Salud UC.

\section{MATERIAL Y MÉTODO}

\section{Pacientes}

Estudio prospectivo entre enero del 2003 y marzo del 2004, de pacientes consultantes al Servicio de Oorrinolaringología de la Red de Salud UG, que presentaron patología vestibular periférica de reciente diagnóstico, con vértigo e inestabilidad de la marcha objetivada mediante examen VII par (audiometría más prueba calóricabitermal a $30^{\circ}$ y $44^{\circ} \mathrm{C}$ ), de edades entre 18 y 70 años, sin distinción por sexo, y que entendieron los objetivos del estudio y firmaron un consentimiento informado. Se excluyeron pacientes en los que se sospechó vértigo central, que no comprendieron los ejercicios indicados, que tenían alguna dificultad locomotora de base o que tomaron antivertiginosos previa mente; los que presentaron vértigo posicional paroxístico benigno (VPPB) más hiporreflexiavestibular unilateral, inicialmente fueron tratados con maniobras de reposición de partículas, y al ya no presentar sintomatología de VPPB, fueron ingresados al estudio.

Se establecieron 2 grupos: grupo intervenido mediantelaaplicación de ejercicios de RV; y grupo control, que recibió tratamiento médico estándar indicado por otorrinolaringólogos del servicio (antivertiginosos ora les en caso de sintomatología intensa).

\section{Método}

Se diseñó una encuesta de rehabilitación vestibular valorada según escala de Likert, de 1 a 4 puntos, equivalente a actividad realizada de forma normal hasta con severa dificultad (Figura 1), extraída y resumida de la encuesta usada por Cohen $\mathrm{IP}^{0}$ en 1991, para determinar el grado de desequilibrio

\begin{tabular}{|l|l|l|l|l|}
\hline Acción & Normal & Leve dificultad & Moderada dificultad & Severa dificultad \\
\hline Caminar & & & \\
Caminar en línea & & & & \\
Acostarse & & & \\
Pararse & & & \\
Trotar & & & \\
Sentarse & & & \\
Grar & & & \\
Bailar & & & \\
Recoger objetos & & & \\
Agacharse & & & \\
Puntaje total y equivalencias: & & & \\
$-\quad \leq 10$ puntos: equilibrio normal. & & & \\
$-\quad 11$ a 20 puntos: desequilibrio leve. &
\end{tabular}

Figura 1. Encuesta de rehabilitación vestibular valorada (normal= 1 punto, hasta severa dificultad= 4 puntos). 
presentado y la alteración de actividades de la vida diaria. Se definieron puntajes y equivalencias: $\leq 10$ puntos igual a equilibrio normal, entre 11 y 20 puntos igual a desequilibrio leve, entre 21 y 30 puntos igual a desequilibrio moderado, y entre 31 y 40 puntos igual a desequilibrio severo.

Luego de aplicada la encuesta a todo el grupo, a los casos se les entregó la pauta de ejercicios de RV (Figura 2), diseñados por
Cawthorne ${ }^{21}$ y Cooksey $^{10}$, con la indicación expresa de ser realizados diariamente, en su domicilio.

Los ejercicios consistieron en:

1) Seguimiento del pulgar: mejora el enfoque de objetos en movimiento.

2) Caminar en tandem y con giros rápidos: mejora coordinación entre las extremidades y el sistema vestibular.

UNIVERSIDAD CATOLICA

UDA OTORRINOLARINGQLOG

OTONEUROLOGA

Programa de Rehabilitación Vestibular

Este programa de ejercicios ha sido diseñado especialmente para mejorar su desequilibrio en la marcha y depende principalmente de Ud., ya que la pauta que le entregamos es para realizar en su hogar, es su responsabilidad practicarla por lo que le solicitamos su esfuerzo y constancia.

\section{I.- Ejercicio de seguimiento del pulgar}

Gire rápidamente su cabeza de lado a lado con los brazos extendidos y la vista fija en sus dedos pulgares. Hágalo durante dos minutos y repita cambiando la dirección del movimiento hacia arriba y abajo.

Este ejercicio ayuda a enfocar objetos mientras su cabeza está en movimiento.

Practíquelo varias veces al día.

\section{II.- Ejercicio de mover la cabeza}

- De pie con los pies separados, realice giros rápidos de su cabeza de derecha a izquierda, arriba y abajo por dos minutos. Descanse dos minutos.

- Sentado mueva la cabeza de atrás hacia delante, luego de hombro a hombro y finalmente realice movimientos rotatorios en $360^{\circ}$ (a derecha y luego a izquierda). Realícelos durante tres minutos.

Esta terapia se indica para habituarse a movimientos rápidos de la cabeza, los que son responsables del desequilibrio momentáneo. Realícela dos veces al día.

\section{III.- Ejercicio de recostarse y pararse}

Sentado en su cama, levántese y acuéstese alternadamente a derecha e izquierda unas diez veces para cada lado. Hágalo dos veces, al levantarse y acostarse.

Esto le ayudará para el entrenamiento del cerebelo, coordinando movimientos rápidos del cuerpo y cabeza.

\section{IV.- Cuerda Floja}

Camine en un pasillo simulando sobre una cuerda floja adelante y atrás varias veces (unos 15 pasos). Este ejercicio le ayudará a mantener un caminar más recto y seguro.

\section{V.- Caminar con giros rápidos}

Camine en un pasillo en línea recta dando siete pasos, gire bruscamente y hágalo en dirección contraria; vuelva a girar y repita por lo menos 10 veces.

Puede realizarlo unas tres veces al día.

Caminará más seguro y podrá efectuar cambios de dirección sin desestabilizarse.

\section{VI.- Pararse con los pies juntos}

Colóquese de pie con los pies bien juntos y trate de mantener esa posición por lo menos durante 15 segundos, al lograr este tiempo cierre los ojos y manténgase allí por lo menos 15 segundos más (sin abrir los ojos). Una vez logrado esto debe realizar el mismo ejercicio sobre una superficie más difícil, como por ejemplo, una alfombra gruesa o sobre una almohada. Haga esto varias veces al día y al comienzo con la ayuda de otra persona para evitar caídas.

Esto lo indicamos para mejorar el equilibrio, usando la información sensorial proveniente de las extremidades inferiores y el oído.

\section{VII.- Ejercicio de recoger botones o monedas}

Coloque 7 botones o monedas en el suelo, cerca de una mesa, Ud. Debe recoger los objetos de uno en uno y depositarlos cada vez sobre la mesa. Practíquelo tres veces seguidas, tratando cada vez de disminuir el tiempo.

\section{VIII.- Lanzando una pelota}

Tome una pelota (primero una grande luego una pequeña) con ambas manos y láncela repetidamente por sobre su cabeza tratando de que no caiga al suelo. Luego tome la pelota y láncela contra una pared, al rebotar, trate de tomarla sin que caiga al suelo.

Esta terapia es importante para la coordinación ojo-manoreflejos motores.

Ante cualquier inquietud puede contactarnos por teléfono.

Figura 2. Programa de rehabilitación vestibular entregado a grupo estudiado. 
3) Rotación de la cabeza en todos los grados: habituación a movimientos cefálicos rápidos.

4) Recostarse hacia ambos lados: mejora la coordinación cerebelosa.

5) Recoger monedas: ejercitación del sistema locomotor.

6) Lanzar el balón: coordinación entre sistema ocular -sistema propioceptivo- aparato locomotor.

Se citó a los casos y controles cada 2 semanas a fin de que los tecnólogos médicos objetivaran el avance logrado en el equilibrio dinámico y estático mediante las pruebas clásicas de Romberg, Untemberger y diferentes tipos de marcha, y constatar así la realización rutinaria de los ejercicios.

En el último control (a las 6 semanas de la primera encuesta), se citó a todos los pacientes para completar nuevamente la encuesta de RV valorada para compararla con la del ingreso al estudio.

\section{Estadística}

Se utilizaron descriptores estadísticos generales, como media y desviación estándar (DE). La comparación entre los resultados de ambos grupos se realizó mediante chi cuadrado. Se consideró un valor $p$ significativo cuando fue $<0,05$. Para calcular la relación entre variables (edad y puntajes), se buscó el coeficiente de correlación.

Los datos fueron analizados mediante el programa SPSS 13.0.

\section{RESULTADOS}

Entre enero de 2003 y marzo de 2004, se reclutaron 48 pacientes. $\mathrm{日}$ grupo control constó de 22 pacientes, cuyo rango de edades fue entre 32 y 70 años, promedio $53 \pm 7,8$ años, de predominio sexo femenino (18/22); el grupo estudiado, que recibió

Tabla 1. Grupo estudiado y grupo control, edad promedio y su distribución por patologías diagnosticadas durante o post RV, según su evolución

\begin{tabular}{|lccc|}
\hline Descripción & Grupo control & Grupo RV & Valor $p$ \\
\hline N & 22 & 26 & \\
Edad (promedio) & $53 \pm 7,8$ & $54 \pm 9,7$ & 0,118 \\
Diagnóstico (n) & & & 0,947 \\
HVU & 11 & 15 & \\
HVB & 3 & 3 & \\
VPPB + HVU & 4 & 5 & \\
Enfermedad Ménière & 2 & 2 & \\
Parálisis vestibular unilateral & 2 & 1 & \\
\hline
\end{tabular}

HVU: hiporreflexia vestibular unilateral. HVB: hiporreflexia vestibular bilateral. VPPB: vértigo postural paroxístico benigno.

Tabla 2. Grado de desequilibrio en grupo estudio y grupo control en el primer control y a las 6 semanas después

\begin{tabular}{|lccccc|}
\hline & \multicolumn{2}{c}{ Grupo control (\%) } & \multicolumn{2}{c|}{ Grupo RV (\%) } \\
Desequilibrio & $1^{\text {er }}$ Control & 6 sem después & $1^{\text {er }}$ Control & 6 sem después \\
\hline Asintomático & 0 & 0 & 0 & 8 \\
Leve & 36 & 50 & 4 & 92 \\
Moderado & 55 & 41 & 58 & 0 \\
Severo & 9 & 9 & 38 & 0,000 & 0 \\
Valor $p$ & & 0,637 & & & 0,000 \\
\hline
\end{tabular}


Tabla 3. Descripción del grupo que recibió RV: edad al momento de la terapia, hallazgo al VIII par, puntajes obtenidos en la encuesta valorada y grado de desequilibrio pre y post RV

\begin{tabular}{|rcccccc|}
\hline Paciente & Edad (años) & Diagnóstico & Puntaje pre RV & Desequilibrio & Puntaje post RV & Desequilibrio \\
\hline 1 & 56 & HVB & 32 & Severo & 14 & Leve \\
2 & 56 & HVB & 20 & Leve & 15 & Leve \\
3 & 62 & HVB & 37 & Severo & 13 & Leve \\
4 & 42 & HVU & 30 & Moderado & 15 & Leve \\
5 & 43 & HVU & 27 & Moderado & 11 & Leve \\
6 & 44 & HVU & 24 & Moderado & 12 & Leve \\
7 & 46 & HVU & 36 & Moderado & 20 & Leve \\
8 & 55 & HVU & 31 & Severo & 15 & Leve \\
9 & 56 & HVU & 23 & Moderado & 12 & Leve \\
10 & 57 & HVU & 40 & Severo & 18 & Leve \\
11 & 58 & HVU & 27 & Moderado & 12 & Leve \\
12 & 59 & HVU & 25 & Moderado & 15 & Leve \\
13 & 61 & HVU & 24 & Moderado & 11 & Leve \\
14 & 61 & HVU & 25 & Moderado & 15 & Leve \\
15 & 63 & HVU & 22 & Moderado & 12 & Leve \\
16 & 63 & HVU & 29 & Moderado & 12 & Leve \\
17 & 63 & HVU & 34 & Severo & 15 & Leve \\
18 & 63 & HVU & 31 & Severo & 16 & Leve \\
19 & 22 & HVU (Ménière) & 23 & Moderado & 11 & Leve \\
20 & 68 & HVU (Ménière) & 40 & Severo & 15 & Leve \\
21 & 58 & Parálisis vestibular unilateral & 33 & Severo & 10 & Asintomático \\
22 & 46 & VPPB + HVU & 24 & Moderado & 10 & Asintomático \\
23 & 50 & VPPB + HVU & 27 & Moderado & 12 & Leve \\
24 & 50 & VPPB + HVU & 28 & Moderado & 15 & Leve \\
25 & 52 & VPPB + HV & 25 & Moderado & 17 & Leve \\
26 & 61 & VPPB + HVU & 32 & Severo & 11 & Leve \\
\hline
\end{tabular}

HVU: hiporreflexia vestibular unilateral. HVB: hiporreflexia vestibular bilateral. VPPB: vértigo postural paroxístico benigno.

RV fue compuesto por 26 pacientes, de predominio sexo femenino (21/26), con rango de edades entre los 22 y 70 años, promedio $54 \pm 9,7$ años, sin diferencias estadísticamente significativas entre ambos grupos.

Se encontraron diversas alteraciones vestibulares en el examen del VII par como: hiporreflexia vestibular unilateral y bilateral, parálisis vestibular, y vértigo postural paroxístico benigno asociado (Tabla 1), sin diferencias estadísticamente significativas entre ambos grupos.

A comparar las encuestas de rehabilitación vestibular valorada, pre y posejercicios (Tabla 2 ), se observa que en el grupo sometido a RV inicialmente $94 \%$ presentó desequilibrio moderado-se- vero; posejercicios, a las 6 semanas, hubo resolución parcial o total de estos síntomas en todos los pacientes, presentando $92 \%$ desequilibrio leve, y el $8 \%$ restante se encontró asintomático (Tabla 3). En el grupo control inicialmente $64 \%$ de los pacientes presentaron desequilibrio moderado-severo; pero al control a las 6 semanas la sintomatología persistió en $50 \%$ del total de los pacientes, el $50 \%$ restante presentó aún desequilibrio leve, y ninguno se recuperó totalmente.

日 desequilibrio inicial entre ambos grupos, y el desequilibrio a las 6 semanas después, también entre ambos grupos, tuvo diferencia estadísticamente significativa; pero al comparar en cada grupo el nivel de desequilibrio encontrado al pri- 
mer control y a las 6 semanas siguiente, sólo se encontró diferencia estadísticamente significativa en el grupo que recibió RV.

Al correlacionar en el grupo que recibió RV la edad con el delta de puntajes entre la encuesta valorada pre y post RV (si a mayor edad, existiría una mayor reducción de los síntomas luego de la $\mathrm{RV}$ ), se encontró un coeficiente de correlación 0,292 , indicando una relación relativamente débil entre las variables.

\section{DISCUSIÓN}

La RV es un programa de ejercicios de aplicación no invasiva, orientado principalmente a la compensación vestibular, es costo efectiva y beneficiosa en pacientes con desequilibrio estático y/o dinámico. Se basa en ejercicios motores-óculo-vestibulares, los cuales fortalecen intensivamente los reflejos vestíbulo-oculomotores, vestíbulo-cerebelosos y vestíbulo-espinales, con lo cual se recobra gran parte de las actividades del diario vivir, lo que es tremendamente valorado, puesto por lo general, el paciente con vértigo siente su vida y actividades diarias truncadas, como también se afecta su esfera anímica, emocional y social.

La RV se realiza en el hogar, diariamente y su evolución es controlada por el especialista (otorrinolaringólogo o tecnólogo médico) tratante. Requiere de un corto período de terapia, generalmente 6 semanas con 1 ó 2 sesiones de control semanales en promedio. Deben ser periódicas y el avance controlado con la realización de pruebas que midan el equilibrio. Es de vital importancia la motivación del paciente y su familia y el compromiso a seguir la pauta entregada, y que no haya una inhibición farmacológica del SNC.

Según los resultados obtenidos, existen diferencias estadísticamente significativas entre ambos grupos, existiendo en el grupo intervenido mejorías parciales en la mayoría de los pacientes y totales en $8 \%$ de éstos, en comparación con el grupo control que recibió tratamiento farmacológico, donde pocos mejoraron y ninguno se recuperó totalmente. Así también existiría una mayor mejoría luego de la RV a mayor edad de los pacientes. En nuestra serie todos los pacientes tuvieron alguna mejoría, por lo que consideramos que los casos en que la RV no logra mejorar la condición del enfermo, se debería en gran parte a que éste no realizó los ejercicios en la forma ni en la frecuencia correcta, puesto los fracasos descritos son infrecuentes.

Si bien los conceptos iniciales de RV se establecen en la década de los 40 con los trabajos de Cawthorne y Cooksey, los recientes avances en el conocimiento de la fisiopatología de la disfunción vestibular, y los nuevos medios de evaluación clínica, vestibular y postural, han introducido el concepto de «programa ajustado» de RV, que consiste en un plan de rehabilitación individualizado para cada caso. Esto no fue aplicado, puesto cremos que los ejercicios indicados sirven para cualquier etiología, por lo cual, en el peor de los casos, un porcentaje de pacientes estarían realizando ejercicios no específicos para su patología, lo cual no les causa ningún perjuicio.

Se debió también validar la encuesta y no sólo valorarla, pero se escogió un procedimiento similar al de Jacobson ${ }^{15}$; si bien existen encuestas similares, éstas son extensas, como la desarrollada por este autor, y generalmente se orientan a evaluar aspectos funcionales, emocionales y físicos del paciente, lo cual va más allá de lo que pretendíamos evaluar (alteración de actividades comunes de la vida diaria, como caminar, agacharse, bailar, etc.).

Fnalmente, consideramos que la RV es una terapia efectiva, y que es importante al momento de iniciar la terapia el recalcarle al paciente que es un método no invasivo, sin medicamentos, ni efectos secundarios, y que luego de realizada la $R V$, existe una alta probabilidad de que mejore además su estado físico y mental.

En nuestro servicio la RV se utiliza con gran éxito; pero nos gustaría realizar a futuro un estudio de mayor envergadura, randomizado y doble ciego a fin de evitar sesgos.

\section{REFERENCIAS}

1. J KATz. «Handbook Of Cinical Audiology». Fourth Edition. Ed: Library Of Congress. Baltimore, 1994.

2. KrognKE K. How Common Are Various Causes Of Dizziness? A Oritical Review. South Med J 2000 Feb; 93(2): 160-7. 
3. A. Cesarani, D. Alpini, B. Monti, and G Raponl. The Treatment $O$ f Acute Vertigo. Neurol Sci 2004 Mar; 25(1): 26-30.

4. Sloane P, Blazrr D, George LK. Dizziness In A Community Ederly Population. J Am Geriatr Soc 1989; 37: 101-8.

5. Drolla H, Penberton J. Vertigo In A Random Sample Of Bderly People Living In Their Homes. J Laryngol Otol 1953; 67: 689-94.

6. BrooklenuRst JC, EXton-Smith AN. Fracture of The Fémur In Od Age: A Two-Centre Study of Associated Cinical Factors And The Cause $O$ f The Fall. Age Ageing 1978; 7: 2-15.

7. L. OKkVIST. «Vertigo». Departamento De O.R.L., Hospital Universitario Linkoping, Suecia 1998.

8. RL. S⿴囗十⺝RSON, GW. CRONIN. «Comparison of the canalith repositioning procedure and vestibular habituation training in forty patients with benign paroxysmal positional vertigo». En: Year book of otolaryngology head and neck surgery. Tomo i. Ed. M. Paparella, r. Holt. U.s.a. 1997.

9. Canthorne T. The Phisiologycal Basis For Head Exercices. J Chartsoc Physiother 1944; 30: 106-7.10.

10. COOKSEY FS. Rehabilitation in Vestibular Injuries. Proc R Socmed 1945; 39: 273-8

11. Dix R, Hallpike CS. The Patology, Symptomatology And Diagnosis of Certain Common
Disorders Of The Vestibular System. Proc $R$ Soc Med 1952; 54: 341-54.

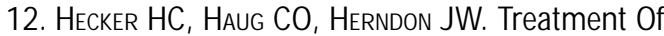
The Vertiginous Patient Using Cawthorne's Vestibular Exercises. Laryngoscope 1974 Nov; 84(11): 2065-72.

13. CoHev H. Measuring The Integrity of The Vestibular System. Am J Occup Ther 1991 Feb; 45(2): 183.

14. WHITNEY SL. Dizziness And Balance Disorders. Cin Management 1991; 11: 42-8.

15. Jacobson GP, NeMman CW. The Development of The Dizziness Handicap Inventory. Arch Otolaryngol Head Neck Sug 1990; 116: 424-7.

16. Fostri CA. Vestibular Rehabilitation. Baillieres Cin Neurol 1994 Nov; 3(3): 577-92.

17. Cohen H, Miller L, KaneWineand M, Hathed C. Vestibular Rehabilitation With Graded $O_{c}$ cupations. Am J Phys Ther 1995; 49: 362-7.

18. KघM RJ, Cook M, MartinI D. Balance Rehabilitation Therapy. Laryngoscope 1992; 102: 1302-7.

19. NT. Shitardd, SA. Telan, M. SMith+Whmook AND A. RAJ. "Vestibular And Balance Rehabilitation Therapy». J Oolaryngol Head Neck Surg 1993.

20. Corev H. Vestibular rehabilitation reduces functional disability. Otolaryngol Head Neck Surg 1992 Nov; 107(5): 638-43.

21. Camthorne T. Vestibular injuries. Proc $R$ Med 1946; 39: 270-3. 